\title{
Proyecto SHE: School and Home Emotions
}

\author{
JosÉ FRANCISCO SANTAS BONA \\ al152927@uji.es \\ NiEVES FuENTES-SÁNCHEZ \\ al262229@uji.es \\ IRENE JAÉN PARRILLA \\ ijaen@uji.es \\ JAHEL MOLINA ULLDEMOLINS \\ al287530@uji.es \\ M. CARMEn PAstor VerchiLI \\ mpastor@uji.es
}

\section{Resumen}

Hoy en día, la inteligencia no garantiza la felicidad en nuestro entorno, trabajo o vida social. El éxito personal y profesional no se vincula únicamente a los conocimientos académicamente adquiridos, sino también al dominio de competencias emocionales, cuyo desarrollo ayuda a gestionar las diferentes situaciones estresantes que se dan en el día a día. En este trabajo se describe un programa en educación emocional (SHE) -diseñado e implementado como estudio piloto-, basado en modelos científicos ampliamente conocidos sobre inteligencia (Salovey \&y Mayer, 1997) y regulación emocional (Gross \&y John, 2003). Mediante dicho programa se pretende poner al alcance de los niños las sinergias creadas por una educación emocional global: niños, profesores y padres. Para ello, se seleccionaron tres muestras de participantes: niños/niñas de $4^{\circ}$ de primaria, padres y madres, y profesorado del mismo centro escolar. La intervención consistió en desarrollar e impartir sesiones bi-quincenales sobre educación emocional. Los resultados mostraron efectos significativos sobre el uso de estrategias de regulación emocional -en la muestra de estudiantes y progenitores-, con una disminución de la supresión emocional y un aumento de la reevaluación cognitiva. En el caso de los profesores, disminuyeron las puntuaciones en afecto negativo. Los valores de funcionamiento familiar permanecieron estables tras la intervención. Por último, no se observaron cambios significativos en las habilidades de inteligencia emocional en ninguno de los tres grupos a estudio.

Palabras clave: inteligencia emocional, regulación emocional, estudiantes, profesorado, progenitores

\section{Abstract}

Today, intelligence does not guarantee happiness in our environment, work or social life. Personal and professional success is not only related to academic knowledge but also to 
the domain of emotional competences, whose development helps to manage the different stressful situations that occur on a daily basis. In this paper we describe a program in emotional education (SHE), designed and implemented as a pilot study, based on well-known scientific models of intelligence (Salovey \&and Mayer, 1997) and emotional regulation (Gross \&and John, 2003). This program aims to make available to children the synergies created by a global emotional education: children, teachers and parents. For this purpose, three samples of participants were selected: children from 4th grade primary, a group of parents of 4 th grade and a group of teachers from the same school. The intervention consisted of developing and imparting bi-fortnightly sessions on emotional education. The results showed significant effects on the use of emotional regulation strategies - in student and parent samples - with a decrease in emotional suppression and an increase in cognitive reevaluation. In the case of teachers, the scores on negative affection decreased. The values of family functioning remained stable after the intervention. Finally, no significant changes in emotional intelligence skills were observed in any of the three study groups

Keywords: emotional intelligence, emotion regulation, students, teachers, parents

\section{Introducción}

«Sólo se aprende bien aquello que se ama»

(Mora, 2015)

¿Por qué educar emociones? En 1946, la Organización Mundial de la Salud definió el concepto de salud como «estado de completo bienestar físico, social y mental, y no sólo asociado a la ausencia de enfermedades». La educación emocional ha surgido en los últimos 25 años como un concepto clave en las relaciones interpersonales en diferentes contextos de la faceta humana, como indicador del ajuste emocional, bienestar personal y éxito laboral (Fernández-Berrocal \& Ruiz Aranda, 2008). La entrada en el siglo XXI nos ha sumergido de lleno en una nueva era de conocimiento necesitada de cambios en la formación de las personas. De forma análoga surge la conocida como sociedad del riesgo. Los cambios socioeconómicos producidos en la segunda mitad del siglo XX han hecho que los antiguos y nuevos riesgos para la salud se agudicen y estén más presentes, de forma especial, en niños y adolescentes (Weissberg, Kumpfer \&y Seligman, 2003). En España fallecieron 30 usuarios de motocicleta en 2015 por no utilizar casco. Del mismo modo, el consumo intensivo de alcohol (con riesgo de producir problemas agudos) se ha incrementado hasta el $13,4 \%$ de la población de 150 más años, y casi 30.000 adolescentes de entre 15 y 19 años quedan embarazadas cada año sin desearlo, mientras que la tasa de abortos entre menores de edad ha ido aumentando hasta situarse en el 13.47 en 2011. Resulta paradójico pensar que este incremento en las conductas de riesgo esté ocurriendo en la llamada «sociedad de la información». Sin embargo, estas cifras tan alarmantes no se deben a una falta de información, sino que más bien se producen por una mala gestión de habilidades emocionales y sociales ante las diversas situaciones y problemas de la vida diaria (Fernández-Berrocal \&y Ramos, 2004). Esta carencia ha hecho que los nuevos paradigmas de formación vuelvan su foco atencional a los programas basados en la educación emocional. De hecho, las dimensiones emocionales, especialmente la reparación o regulación emocional, han mostrado una asociación positiva con la satisfacción vital 
(Rey, Extremera, Pacheco \&y Pena, 2011). En un entorno en el que la crisis económica y política ha planteado la necesidad de reordenar la escala de valores, una educación socialmente responsable se antoja necesaria para asegurar el bienestar de la sociedad del futuro. Se necesitan personas con competencias sociales y emocionales, que sepan trabajar en equipo, resolver conflictos, que sean creativas, que aprendan a tener una vida satisfactoria. $Y$ para aprender, la emoción y la educación emocional, son la llave de la atención, puerta de la curiosidad, principio del aprendizaje (Mora, 2015).

Precisamente, el objetivo del presente trabajo es diseñar e implementar un programa de educación emocional que mejore la calidad en la educación, tomando para ello una muestra de niños y niñas de $4^{\circ}$ de primaria. Así, pretendemos proporcionar estrategias de regulación emocional comunes a los tres colectivos (alumnado, profesorado y progenitores), crear espacios donde poder reconocer y expresar emociones y favorecer el diálogo sobre los estados afectivos de cada persona. Aprovechar las sinergias creadas por una educación análoga a niños/niñas, profesorado y padres/madres será la principal singularidad que cabe destacar como principal aportación de este trabajo.

\section{Método}

\section{Participantes}

La muestra experimental del presente estudio fue obtenida a través de la colaboración con el CEIP José Soriano de Vila-real (Castellón). La muestra se dividió en tres grupos: (1) niños/niñas de $4^{\circ}$ de primaria, (2) padres/madres de $4^{\circ}$ de primaria, y (3) profesorado del centro escolar. La muestra final de estudiantes estuvo compuesta por 55 niños (28) y niñas (27), con un rango de edad entre 9 y 10 años (Media $=9.5$ años). Respecto a la muestra de profesorado que participó en el programa fue de 20 personas (19 mujeres y 1 hombre), con un rango de edad entre 27 y 48 años (Media $=33,4$ ). En cuanto a la muestra de progenitores que participaron en el programa, finalmente contamos con un total de 21 ( 3 padres, 18 madres), con un rango de edad entre 41 y 50 años (Media $=44,67$ ).

\section{Instrumentos}

Trait Meta-Mood Scale (TMMS-24; Fernández-Berrocal, Extremera \&y Ramos, 2004; Salovey, Mayer, Goleman, Turvey \&y Palfai, 1995). Esta escala proporciona información sobre el nivel de Inteligencia Emocional a partir de tres subescalas: Atención a los sentimientos, Claridad de los sentimientos y Reparación del estado de ánimo. La escala contiene 24 ítems, con una respuesta tipo Likert de 5 puntos (1 Nada de acuerdo - 5 Totalmente de acuerdo).

Emotion Regulation Questionnaire (ERQ; Cabello, Salguero, Fernández-Berrocal \&y Gross, 2013; Gross \&y John, 2003). Evalúa dos estrategias de regulación emocional: la reevaluación cognitiva y la supresión emocional. Los participantes deben indicar su grado de acuerdo con las afirmaciones utilizando una escala Likert de 7 puntos ( 1 = totalmente en desacuerdo, 7 = totalmente de acuerdo). En nuestro caso, decidimos mantener la versión que contiene una escala Likert de 5 puntos ( $1=$ totalmente en desacuerdo, $5=$ totalmente de acuerdo) para poder comparar resultados con los obtenidos con el ERQ-CA aplicado a los niños.

Emotional Regulation Questionnaire for Children \& Adolescents (ERQ-CA; Gullone \&y Taffe, 2012). (Pastor et al., en preparación). Versión en castellano del cuestionario ERQ para niños y adolescentes. 
Positive and Negative Affect Schedule (PANAS; Robles \&y Páez, 2005). Mide afecto y afecto negativo. Utiliza 20 ítems, 10 de los cuales miden el afecto positivo y los 10 restantes afecto negativo. Estos 20 ítems son palabras o adjetivos que describen diferentes sentimientos y emociones. Las califica la persona, según el grado en que las experimenta, en una escala de cinco puntos, en donde 1 significa «muy poco o nada», y 5 «extremadamente».

Escala PANAS-N (Sandín, 2003; Joiner, Sandín, Chorot, Lostao \&y Marquina, 1999; Watson, Clark \&y Tellegen, 1988): análogamente versión para niños y adolescentes.

Behaviour Assesment System of Children S2 (BASC-S2; González, Fernández, Pérez \&y Santamaría, 2004; Reynolds \&y Kamphaus, 1992). Autoinforme de personalidad para niños de 8-11 años que consta de 146 enunciados que han de ser contestados como verdadero o falso. Son cinco las dimensiones que lo conforman: Inadaptación escolar, Desajuste clínico, Adaptación personal, Otros problemas y un índice global, el índice de síntomas emocionales (ISE).

Behaviour Assesment System of Children P2 (BASC-P2; González, Fernández, Pérez \&y Santamaría, 2004; Reynolds \&y Kamphaus, 1992). Evalúa conductas adaptativas y problemáticas en los ámbitos familiar y comunitario, informado por los padres. Está compuesto por 134 ítems de elección entre cuatro alternativas que evalúan 6 dimensiones: Exteriorizar Problemas, Interiorizar Problemas, Problemas Escolares, Otros Problemas, Habilidades Adaptativas y el Índice de Síntomas Comportamentales (ISC).

Family Adaptability and Cohesión Evaluation Scale (FACES 20-esp; Martínez-Pampliega, Iraurgi, Galíndez \&y Sanz, 2006). Escala breve (20 ítems) de análisis de funcionamiento familiar a partir de la escala americana Family Adaptability and Cohesion Evaluation Scale (FACES II). Recoge las dimensiones de Cohesión y Adaptabilidad del modelo circumplejo (Olson, 1988).

Escala de Satisfacción Laboral Versión para Profesores (ESL-VP; Anaya, 2005). Recoge en 44 ítems información sobre las facetas que los profesores consideran importantes para la satisfacción laboral y el grado en el que estas facetas se encuentran presentes en su trabajo actual. Este modelo se estructura alrededor de cinco dimensiones principales: diseño del trabajo, condiciones de vida asociada al trabajo, realización personal, promoción y superiores.

\section{Procedimiento}

La formación en educación emocional fue impartida a los tres grupos experimentales. Se estructuró en siete sesiones de 50 minutos de duración en el caso de los niños, y nueve sesiones de 60 minutos para el profesorado y progenitores. Las sesiones consistían en talleres teórico-prácticos en los que se impartían contenidos utilizando el juego como herramienta principal. Las sesiones fueron bi-quincenales, coincidiendo temporalmente la temática impartida en los tres grupos. El inicio de los talleres tuvo lugar a inicios de enero de 2016, finalizando en junio de ese mismo año. La evaluación del programa de educación emocional que se describe en el presente trabajo se realizó en dos momentos: pre-intervención -previamente a la implementación del programa- y post-intervención -última de las sesiones impartidas para cada muestra. En el presente estudio se evaluaron diferentes variables afectivas -Inteligencia Emocional Percibida, Estrategias de Regulación Emocional y Afecto Positivo y Negativo-, de clima familiar -Adaptabilidad y Cohesión-, y sobre la satisfacción laboral del profesorado (ver Figura 1). En la última sesión, algunos instrumentos (BASC-S2, BASC-P2) tuvieron que ser reemplazados por otros (TMMS, PANAS) para reducir el número de ítems y, con ello, el tiempo de administración. 


\begin{tabular}{ccc}
\hline & PRE & POST \\
\hline \multirow{4}{*}{ NIÑOSIAS } & BASC S2 & PANAS-N \\
& TMMS-24 & TMMS-24 \\
& ERQ_CA & ERQ_CA \\
\hline \multirow{4}{*}{ PROFESORADO } & PANAS & PANAS \\
& TMMS 24 & TMMS 24 \\
& ERQ & ERQ \\
& ESL-VP & ESL-VP \\
\hline \multirow{3}{*}{ PROGENITORES } & BASC-P2 & \\
& FACES I & PANAS \\
& ERQ & FACES ॥ \\
& ERQ \\
& & TMMS 24 \\
\hline
\end{tabular}

Figura 1. Evaluación de la intervención (programa de educación emocional).

\section{Análisis estadístico}

En primer lugar, se calcularon los estadísticos descriptivos (medias y desviaciones típicas) de las diferentes medidas de autoinforme utilizadas en el presente estudio (ERQ/ERQCA, TMMS-24, PANAS/PANAS-N, ESL-VP, FACES-II), por separado para cada una de las muestras experimentales (estudiantes, progenitores y profesorado). En segundo lugar, se realizaron ANOVAS de un factor (Pre- vs. Post-intervención) para analizar si había diferencias en los resultados obtenidos en los diferentes instrumentos entre la primera (pre) y la última sesión (post) del programa de educación emocional. Los análisis fueron realizados con el paquete estadístico IBM SPSS Statistics v.21 y el software JMP 9.0.1.

\section{Resultados}

En las Tablas 1, 2 y 3 se resumen los estadísticos descriptivos para las tres muestras incluidas en nuestro estudio. Para la muestra de estudiantes, los valores obtenidos son comparables a los obtenidos en trabajos anteriores, así como en la validación de los cuestionarios utilizados en este trabajo (Cabello et al., 2013; Fernández- Berrocal et al., 2004; Sandin, 2003). No obstante, las medias obtenidas en las subescalas Supresión Emocional $(12,44)$ y Reevaluación Cognitiva $(23,62)$ del ERQ-CA, así como las subescalas de Atención (3.57), Claridad (3.80) y Reparación emocional del TMMS-24 (3.91) fueron ligeramente más elevados que los obtenidos en las validaciones de estos instrumentos (Medias $=10,49$ y 21,53, respectivamente; Medias $=3,24,3,24$ y 3,30, respectivamente). Por su parte, la media en la dimensión de Afecto Negativo del PANAS (21.2) fue ligeramente inferior comparada con la obtenida en la validación $(18,03)$.

Respecto a la muestra de profesorado, los resultados de nuestro trabajo son totalmente comparables a los de investigaciones anteriores, al igual que en los estudios realizados para 
validar dichos cuestionarios (Gross \& John, 2003; Fernández- Berrocal et al., 2004; Robles \& Páez, 2003). Aun así, cabe resaltar que los promedios obtenidos en las subescalas de Supresión Emocional $(2,14)$ y Reevaluación Cognitiva $(3,44)$ del ERQ fueron ligeramente inferiores a los obtenidos en las validaciones de este instrumento (Medias $=3,15$ y 4,85). Por el contrario, la media obtenida en la subescala de Afecto Negativo del PANAS $(2,60)$ resultó ligeramente superior a la de la validación $(18,03)$.

En el caso de la muestra de progenitores, los valores son equiparables a los obtenidos en trabajos anteriores, así como en la validación de los cuestionarios utilizados en este estudio (Gross \& John, 2003; Fernández- Berrocal et al., 2004; Anaya \& Suárez, 2007; MartínezPampliega et al., 2006). No obstante, las medias obtenidas en las subescalas Supresión Emocional $(2,48)$ y Reevaluación Cognitiva $(3,49)$ del ERQ resultaron ligeramente inferiores a las obtenidas en las validaciones de este instrumento (Medias $=3,15$ y 4,85, respectivamente). Finalmente, se observó que la media en la subescala de Cohesión del FACES II $(4,29)$ resultó superior al valor obtenido en la validación del instrumento $(3,63)$.

En cuanto a los ANOVAs realizados, puede observarse una mejora en la regulación emocional de los estudiantes tras la intervención realizada (ver Tabla 1). Concretamente, el uso de estrategias de Supresión Emocional descendió significativamente para la muestra de estudiantes, acompañado de un aumento en la utilización de estrategias de Reevaluación Cognitiva. Una tendencia similar pudo apreciarse en el caso de los progenitores por lo que se refiere al uso de Reevaluación Cognitiva, mientras que no se encontraron diferencias estadísticamente significativas para la muestra de profesorado (ver Tabla 3). Respecto a las habilidades vinculadas con la inteligencia emocional (Atención, Claridad y Reparación Emocional), no se han observado diferencias significativas entre los valores pre- y post-intervención. En relación a los profesores (ver Tabla 2), se encontró una disminución significativa del Afecto Negativo al finalizar la intervención. Igualmente, se observó una tendencia a la disminución del Afecto Positivo, acompañada de disminuciones significativas en las subescalas Diseño del Trabajo, Realización personal y Promoción post-intervención. Por último, no se encontraron diferencias estadísticamente significativas en cuanto a la percepción del clima familiar por los progenitores.

Tabla 1

Medias, desviaciones típicas y ANOVAS (Pre vs. Post-intervención) de los autoinformes ERQ-CA, TMMS 24 y PANAS-N, para la muestra de estudiantes $(N=55)$

\begin{tabular}{|c|c|c|c|c|c|c|}
\hline & \multicolumn{2}{|c|}{ PRE } & \multicolumn{2}{|r|}{ POST } & \multicolumn{2}{|c|}{ ANOVA } \\
\hline & $\mathrm{N}$ & Media (DT) & $\mathrm{N}$ & Media (DT) & $F$ & $p$ \\
\hline \multicolumn{7}{|l|}{ ERQ-CA } \\
\hline Supresión & 54 & $12,44(3,89)$ & 49 & $10,94(3,99)$ & 3,76 & ,055 \\
\hline Reevaluación & 54 & $23,62(4,08)$ & 49 & $24,73(4,51)$ & 3,68 & ,058 \\
\hline \multicolumn{7}{|l|}{ TMMS 24} \\
\hline Atención & 55 & $28,53(5,73)$ & 50 & $26,34(5,94)$ & 3,68 &, 06 \\
\hline Claridad & 55 & $30,44(5,32)$ & 50 & $29,86(6,35)$ &, 25 &, 61 \\
\hline Reparación & 55 & $31,33(5,53)$ & 50 & $31,64(6,02)$ & ,08 &, 78 \\
\hline \multicolumn{7}{|l|}{ PANAS-N } \\
\hline Afecto Positivo & - & - & 50 & $24,38(3,10)$ & - & - \\
\hline Afecto Negativo & - & - & 50 & $18,08(3,03)$ & - & - \\
\hline
\end{tabular}


Tabla 2

Medias, desviaciones típicas y ANOVAS (Pre vs. Post-intervención) de los autoinformes ERQ, PANAS, TMMS-24, ESL-VP, para la muestra de profesores $(N=20)$

\begin{tabular}{|c|c|c|c|c|c|c|}
\hline & \multicolumn{2}{|r|}{ PRE } & \multicolumn{2}{|r|}{ POST } & \multicolumn{2}{|c|}{ ANOVA } \\
\hline & $\mathrm{N}$ & Media (DT) & $\mathrm{N}$ & Media (DT) & $F$ & $p$ \\
\hline \multicolumn{7}{|l|}{ ERQ } \\
\hline Supresión & 20 & $8,55(3.20)$ & 13 & $9,08(3,20)$ & ,21 & ,647 \\
\hline Reevaluación & 18 & $20,61(4.41)$ & 13 & $20,77(3,30)$ & 01 & ,914 \\
\hline \multicolumn{7}{|l|}{ TMMS 24} \\
\hline Atención & 20 & $26,85(5.80)$ & 13 & $25,23(5,21)$ & 67 & ,421 \\
\hline Claridad & 20 & $27,65(3.73)$ & 13 & $26,69(6,36)$ & ,30 &, 588 \\
\hline Reparación & 20 & $26,20(5.91)$ & 13 & $26,38(6,21)$ & .01 & .932 \\
\hline \multicolumn{7}{|l|}{ PANAS-N } \\
\hline Afecto Positivo & 20 & $35,90(5.06)$ & 13 & $31,67(6,87)$ & 3,64 & ,066 \\
\hline Afecto Negativo & 20 & $25,60(5.48)$ & 13 & $20,46(5,82)$ & 6,60 &, 015 \\
\hline \multicolumn{7}{|l|}{ ESL-VP } \\
\hline Diseño de trabajo & 20 & $41,85(6.06)$ & 13 & $36,23(6,87)$ & 6,10 & ,019 \\
\hline Condiciones de vida & 20 & $24,65(5.76)$ & 13 & $21,54(5,16)$ & 2,50 & , 125 \\
\hline Realización personal & 20 & $28,15(4.88)$ & 13 & $23,69(4,63)$ & 6,84 & 014 \\
\hline Promoción & 20 & $13,10(3.99)$ & 13 & $8,62(4,98)$ & 8,20 &, 007 \\
\hline Salario & 20 & $6,35(1.35)$ & 13 & $6,62(1,45)$ & 0,29 & ,595 \\
\hline
\end{tabular}

Tabla 3

Medias, desviaciones típicas y ANOVAS (Pre vs. Post-intervención) de los autoinformes ERQ, PANAS, TMMS-24, FACES II, para la muestra de progenitores $(N=18)$

\begin{tabular}{|c|c|c|c|c|c|c|}
\hline & \multicolumn{2}{|r|}{ PRE } & \multicolumn{2}{|r|}{ POST } & \multicolumn{2}{|c|}{ ANOVA } \\
\hline & $\mathrm{N}$ & Media (DT) & $\mathrm{N}$ & Media (DT) & $F$ & $p$ \\
\hline \multicolumn{7}{|l|}{ ERQ } \\
\hline Supresión & 18 & $9,90(0.56)$ & 9 & $10,00(0.84)$ & 01 & ,92 \\
\hline Reevaluación & 18 & $20,94(1.06)$ & 9 & $23,11(1.50)$ & 1,40 &, 25 \\
\hline \multicolumn{7}{|l|}{ TMMS 24} \\
\hline Atención & - & - & 9 & $24,67(6,42)$ & - & - \\
\hline Claridad & - & - & 9 & $22,56(4,00)$ & - & - \\
\hline Reparación & - & - & 9 & $28,78(4,06)$ & - & - \\
\hline \multicolumn{7}{|l|}{ PANAS-N } \\
\hline Afecto Positivo & - & - & 9 & $31,44(6,08)$ & - & - \\
\hline Afecto Negativo & - & - & 9 & $21,11(4,62)$ & - & - \\
\hline \multicolumn{7}{|l|}{ FACES II } \\
\hline Cohesión & 21 & $4,29(0,10)$ & 9 & $4,22(0,15)$ & 14 &, 71 \\
\hline Adaptabilidad & 21 & $3,68(0,10)$ & 9 & $3,64(0,16)$ & ,03 & 87 \\
\hline
\end{tabular}




\section{Discusión y conclusiones}

El presente estudio pretendía observar los cambios producidos tras una intervención en estudiantes de $4^{\circ}$ de primaria, progenitores y profesores. Aunque los resultados fueron los esperados para la muestra de estudiantes, no se cumplieron las hipótesis planteadas para las muestras de profesores y progenitores. A este respecto, cabe mencionar que el programa de educación emocional tuvo una reducción en el número de horas de formación inicialmente planteadas, pudiendo ser insuficientes para poder observar resultados significativos, tal y como se han obtenido en otros programas (Ruiz-Aranda et al., 2008; Repetto, 2009). No obstante, nuestros resultados apoyan parcialmente la evidencia experimental previa (Fernández Berrocal et al., $2005,2008)$. En cuanto al afecto negativo, la disminución observada en la muestra de profesores estaría de acuerdo con la literatura revisada (Cabello et al., 2010; Fernández Berrocal et al., 2005). Esta disminución podría ser una consecuencia de la formación adquirida en el programa SHE. No obstante, se encontró una importante reducción en afecto positivo para la muestra de profesorado que podría explicarse por el complicado momento en el que se realizó la evaluación post-intervención. Creemos que podría haberse dado una contaminación de los resultados ya que esta recogida de datos coincidió con el final del curso académico, y con la repentina implantación del Plan de Xarxa Llibres. Esta sobrecarga de trabajo afectó seriamente a la disponibilidad de los docentes que participaron voluntariamente en el programa SHE y generó un incremento en sus niveles de estrés. Dicha situación escolar podría igualmente explicar la disminución en los resultados de satisfacción laboral post-intervención (Extremera et al., 2003). En cuanto al funcionamiento familiar, no se observaron variaciones significativas en los niveles de cohesión $y$ adaptabilidad tras la intervención realizada.

En general, el hecho de que las diferencias pre- vs. post-intervención hayan sido marginalmente significativas en nuestro trabajo podría deberse a distintas limitaciones. En primer lugar, al reducido tamaño de la muestra experimental, sobre todo en los grupos de progenitores y profesorado. En segundo lugar, podría deberse a que los instrumentos utilizados no han sido suficientemente sensibles para captar el cambio emocional inducido por el programa diseñado e implementado en el presente estudio. En tercer lugar, podría explicarse a partir del número de sesiones puesto que podría ser insuficiente para notar un cambio significativo, tal y como hemos comentado anteriormente. Debido a la propia dinámica del curso académico, el número total de sesiones fue claramente inferior a lo previsto en el diseño original del programa. En este sentido, debemos considerar su implementación como un estudio piloto, pero entendemos que sería necesaria una intervención más prolongada en el tiempo para poder obtener cambios significativos. En cuarto lugar, la implantación del programa SHE en el horario lectivo del centro escolar ha supuesto un importante handicap para cumplir con el calendario establecido a priori. De hecho, el retraso de varias sesiones al principio del programa hizo que la administración post-intervención de los instrumentos coincidiera con el final del curso académico. Este momento pudo haberse visto contaminado por la presencia de elevados niveles de estrés, no sólo en el profesorado sino también en los propios estudiantes. A este respecto, se podría haber introducido algún instrumento para evaluar el estrés percibido en las diferentes muestras y cuantificar este efecto no previsto inicialmente, ayudando a obtener una visión más objetiva del impacto del programa en educación emocional. 


\section{Referencias bibliográficas}

Cabello, R., Fernández-Berrocal, P., Gross, J. J., \& Salguero, J. M. (2013). Spanish adaptation of the Emotion Regulation Questionnaire. European Journal of Psychological Assesment, 29(4), 234-240.

Extremera, N. \& Fernández-Berrocal, P. (2003b). La inteligencia emocional en el contexto educativo: hallazgos científicos de sus efectos en el aula. Revista de Educación, (332), 97-116

Fernández-Berrocal, P., Extremera, N. \& Ramos, N. (2004) Validity and realibility of the spanish modified version of the trait meta-mood scale. Psychological Reports, (94), 751-755.

Fernández-Berrocal, P. \& Extremera, N. (2005). La Inteligencia Emocional y la educación de las emociones desde el Modelo de Mayer y Salovey. Revista Interuniversitaria de Formación Del Profesorado, 19(3), 63-94.

Fernández-Berrocal, P. \& Ruiz Aranda, D. (2008). La inteligencia emocional en la educación. Electronic Journal of Research in Educational Psychology 6 (15), 421-436.

González, J., Fernández, S., Pérez, E. \& Santamaría, P. (2004). Spanish adaptation of Behavior Assessment System for Children and Adolescents: BASC. Madrid: TEA Ediciones.

Gross, J. J. \& John, O.P. (2003). Individual differences in two emotion regulation processes: Implications for affect, relationships, and well-being. Journal of Personality and Social Psychology, (85), 348-362.

Martínez-Pampliega, A., Iraurgi, I., Galíndez, G. \& Sanz, M. (2006). Family Adaptability and Cohesion Evaluative Scale (FACES): desarrollo de una versión de 20 ítems en español. International Jounal of Clinical and Health Psychology, 6, 2, 317-338.

Mayer, J. D. \& Salovey, P. (1997). What is emotional intelligence? Eln P. Salovey \& D. J. Sluyter (Eds.), Emotional development and emotional intelligence: Educational implications (pp. 3-34). New York: Harper Collins.

Olson, D. H. (1988). Capturing family change: Multi-system level assesment. En L. C. Wynne (Ed.), The state of the art in family therapy research: controversies and recommendations. (pp. 75-80). Nueva York: Family process press.

Pastor, M. C., López-Penadés, R., Cifre, E. \&Y Moliner- Urdiales, D. (en preparación). Response-focused regulation of emotions is associated with psychological maladjustment: Evidence from a Spain Adolescent Sample.

Repetto, E. (2009) Formación en competencias Socioemocionales. Ed. La Muralla, Madrid.

Rey, L., Extremera-Pacheco, N. \& Pena, M. (2011). Perceived Emotional Intelligence, Self-Esteem and Life Satisfaction in Adolescents. Psychosocial Intervention, 20(2), 227-234.

Reynolds, C. R. \& Kamphaus, R. W. (1992). Sistema de Evaluación de la Conducta de niños $y$ adolescentes (BASC). Madrid: TEA.

Robles R. \& Páez F. (2003). Estudio sobre la traducción al español y las propiedades psicométricas de las escalas de afecto positivo y negativo (PANAS). Salud Mental. 26. $\mathrm{n}^{\circ} 1$.

Ruiz-Aranda, D., Fernández-Berrocal, P., Cabello, R. \& Salguero, J. M. (2008). Educando la Inteligencia emocional en el aula: Proyecto Intemo. Electronic Journal of Research in Educational Psychology, (6), 240-251.

Sandín, B., Chorot, P., Lostao, L., Joiner, T. E., Santed, M. A. \& Valiente, R. M. (1999). Escalas Panas de afecto positivo y negativo: validación factorial y convergencia transcultural. Psicothema, (11), 37-51.

Sandín, B. (2003). Escalas PANAS de Afecto Positivo y Afecto Negativo para niños y adolescentes (PANASN). Revista de Psicopatología y Psicología Clínica, (8), 173-182. 
Watson, D., Clark, L. A. \& Tellegen, A. (1988). Development and validation of brief measures of positive and negative affect: The PANAS scales. Journal of Personality and Social Psychology, 54, 1063-1070.

Weissberg, R. P., Kumpfer \& Seligman, M. E. (2003). Prevention that works for children and youth: An introduction. American Psychologist, (58), 425-432. 